\title{
Prognostic value of peritumoral heat-shock factor-1 in patients receiving resection of hepatocellular carcinoma
}

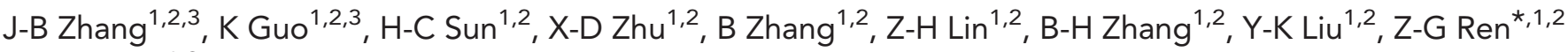 \\ and J Fan ${ }^{\star}, 1,2$ \\ ${ }^{1}$ Liver Cancer Institute and Zhongshan Hospital, Fudan University, 136 Yi Xue Yuan Road, Shanghai, China and ${ }^{2}$ Key Laboratory of \\ Carcinogenesis and Cancer Invasion, Ministry of Education, 136 Yi Xue Yuan Road, Shanghai, China
}

\begin{abstract}
Background: The cross-talk of hepatocellular carcinoma (HCC) cells and abnormal metabolic signals in peritumoral microenvironment modifies our knowledge of hepatocarcinogenesis. As an indispensable modulator of various stresses, the clinical significance of heat-shock transcription factor-1 (HSF1) in HCC microenvironment has never been defined.
\end{abstract}

Methods: Hepatocellular carcinoma and matched peritumoral liver tissues $(n=332)$ were semiquantitatively analysed for HSF1 expression, followed by correlation with clinicopathological parameters (patient outcomes). Moreover, the effects of HSF1 deficiency in L02 on monocarboxylate transporter-4 (MCT4) and HCC cells' colonisation and proliferation were investigated.

Results: High expression of HSF1 in peritumoral tissue but not in HCC tissue was associated with poorer overall survival (OS) and time to recurrence (TTR), especially early recurrence (ER), which was further reconfirmed in validation cohort. Multivariate analysis showed that prognostic performance of peritumoral HSF1 was independent of other clinicopathological factors (hazard ratio for $\mathrm{OS}=2.60, P=0.002$, for TTR $=2.52, P<0.001$ ). Notably, downregulation of HSF1 in L02 decreased MCT4 expression significantly. The supernatant from L02-shRNA-HSF1 in hypoxia, NOT normoxia condition, inhibited HCC cell colonisation and proliferation. Moreover, the combination of peritumoral HSF1 and MCT4 was the best predictor for ER and OS.

Conclusion: High peritumoral HSF1 expression can serve as a sensitive 'readout' for high-risk HCC ER, and could be a potential metabolic intervention target following curative resection.

Hepatocellular carcinoma (HCC) is the fifth most common cancer in men and the seventh in women, with an estimated 749000 new cases and 695000 people projected to die from HCC worldwide in 2008 (Ferlay et al, 2010). The 5-year survival rate of HCC is still very poor despite aggressive conventional therapy like radical resection because most cases of HCC are concomitant with chronic liver disease (e.g. cirrhosis) (Thorgeirsson and Grisham, 2002; Tang et al, 2004). Such non-malignant liver tissues surrounding HCC tissue have a precancerous change where the normal structure of the liver is distorted and its function is impaired (Alison et al, 2011; Tinkle and Haas-Kogan, 2012). As we know, fibrogenesis induces intrahepatic shunts, a barrier between the sinusoids and the hepatocytes, and finally leads to hypoxia (Severi et al, 2010; Nahleh et al, 2012), which has been involved in metabolic reprogramming of cells and leads cancer cells to upload lactate contributing to the acidic microenvironment (Palazon et al, 2012). Moreover, metabolic changes driven by the tumour microenvironment also confer a selective advantage for survival and proliferation of cancer cells (Jin et al, 2011). During the process, cells adapt to chronic stress in the tumour microenvironment by inducing the expression of heat-shock proteins (HSPs) (Calderwood et al, 2006; Luk et al, 2006). As the primary regulator of HSPs, heat-shock factor-1 (HSF1) controls cellular response to stress through a very highly conserved protective mechanism

*Correspondence: Dr J Fan; E-mail: fan.jia@zs-hospital.sh.cn or Dr Z-G Ren; E-mail: ren.zhenggang@zs-hospital.sh.cn

${ }^{3}$ These authors contributed equally to this work

Received 16 April 2013; revised 29 July 2013; accepted 30 July 2013; published online 3 September 2013

(c) 2013 Cancer Research UK. All rights reserved 0007-0920/13 
(Dai et al, 2007; Whitesell and Lindquist, 2009). Recent evidence indicates that HSF1 extends far beyond the classical induction of HSPs and may promote malignant transformation by orchestrating a network of core cellular functions, including proliferation, protein synthesis, glucose metabolism (Whitesell and Lindquist, 2009) and survival in a premalignant microenvironment like hypoxia (Hahn et al, 2004; Brahimi-Horn and Pouyssegur, 2007; Sherman and Multhoff, 2007). Owing to the metabolic shift from oxidative phosphorylation to glycolysis and lactate acid production, hypoxia microenvironment favours the adaptive survival of cancer cells (Brahimi-Horn et al, 2011). Meanwhile, to avoid intracellular acidification, cancer cells must extrude excess lactic acid through lactate transporter (Huber et al, 2010). Monocarboxylate transporter-4 (MCT4), a proton-coupled lactate transporter, is preferentially expressed in cancer cells (Pertega-Gomes et al, 2011; Vander Heiden, 2011), thereby allowing the maintenance of high glycolytic rates through lactate efflux (Halestrap and Wilson, 2012).

As conveyed above, HSF1 enhances longevity and assists cells dealing with stressful attack. By increasing the likelihood of mutations and facilitating malignant cellular adaptation, and thus enabling premalignant cells to reach the essential hallmarks of cancer (Sherman and Multhoff, 2007), HSF1 may contribute to oncogenesis. On the other hand, HSF1 may also be involved in regulating the development of a stress microenvironment (Dai et al, 2007). Recently, it has been found that HSF1 regulates hepatic steatosis and metabolism, making it a key determinant of HCC development. However, the clinical significance of HSF1 in HCC microenvironment remains elusive.

In this study, we investigated expressions of HSF1 in two independent cohorts with a total of 332 resected HCC samples and corresponding peritumoral liver tissues. Our results indicated that high expression of HSF1 in peritumoral tissue but not in HCC tissue was associated with poor patient OS and time to recurrence (TTR) after curative resection, especially for ER. Furthermore, in vitro experiments showed that the supernatant from L02-shRNA-HSF1 cells in hypoxia but not normoxia condition significantly inhibited colony formation and proliferation of HCC cells. This suggests that in the stressful condition, the interplay of surrounding liver cells and HCC cells is more prone to HSF1 dependence. Moreover, downregulation of HSF1 in human liver cell L02 significantly decreased the expression of MCT4, and in tissue microarray analysis, it was shown that the expression of peritumoral HSF1 was significantly correlated with peritumoral MCT4. Through receiver operating characteristic (ROC) curve analysis, we also found that the combination of HSF1 and MCT4 was the best predictor for OS. Altogether, HSF1 may serve as a potential predictor for early recurrence (ER) after HCC curative resection and a novel metabolic target of adjuvant therapy in HCC.

\section{MATERIALS AND METHODS}

Patient and specimen information. We used archival formalinfixed, paraffin-embedded paired tumour with corresponding peritumoral liver tissues from 105 and 227 consecutive HCC patients. These patients were diagnosed with HCC pathologic stages I to IIIa (according to the 2009 International Union Against Cancer Tumour Node Metastasis Classification System, 7th edition) at the Fudan University and Liver Cancer Institute in the period from January 1999 to December 2006 and were randomly enrolled as described (Zhu et al, 2008). Approval was received from the Zhongshan Hospital Research Ethics Committee and written consent was obtained from each patient. The Scheuer system was applied in 100 patients - the surrounding liver tissue was not adequate to score in five patients - for grading and staging of peritumoral liver tissue (Brunt, 2000). The clinicopathological features of these patients are seen in Supplementary Table S1.

None of the patients in our study received radiotherapy or chemotherapy before surgery, and patients were followed until 31 March 2010. The longest follow-up was 118 months, and the median follow-up 65 months in cohort 1 and 44 months in the validation cohort. Follow-up procedures were described in our previous study (Zhu et al, 2008). Patients were briefly monitored by serum $\alpha$-fetoprotein (AFP), abdominal ultrasonography and chest radiography with an interval of 2-6 months according to the postoperative time. Enhancement computed tomography scanning or magnetic resonance imaging was performed every 6-12 months or suspicious recurrence. Combined treatment modalities after recurrence were administered according to a uniform guideline. Overall survival or TTR was defined as the interval between surgery and death or recurrence. If recurrence was not diagnosed, patients were censored on the date of death or the last follow-up.

Immunohistochemistry. The immunohistochemistry protocols and the method of constructing tissue microarray are described elsewhere (Zhu et al, 2008). Primary antibodies were HSF1 (1:200, monoclonal; Cell Signaling Technology, Danvers, MA, USA) and MCT4 (1:200, polyclonal; Santa Cruz Biotechnology, Santa Cruz, CA, USA). Before they were used on the arrays, the antibodies were titrated against normal controls and the concentrations determined to give optimal sensitivity and specificity in the control tissue. Negative controls were treated identically but with the primary antibodies omitted (Supplementary Figure S1).

Scoring of immunohistochemistry and selection of cutoff score for the Kaplan-Meier survival analysis. Under light microscopy, the entire core was analysed at low and high power for each case. Labelling scores were given based on the staining intensity $(0-3$, $0=$ negative, $1=$ weak, $2=$ moderate, $3=$ strong -representative figures are shown in Supplementary Figure S2) and percentage of cells stained (0-5: 0, 1-10, 11-25, 26-50, 51-75 and 76-100\%). The two components were multiplied for an overall staining score semiquantitatively for positive staining as published previously (Bremnes et al, 2002) by three researchers who were blinded to prior knowledge of clinical and pathological parameters and follow-up data. Their results were in complete agreement in $82 \%$ of the cases. In case of disagreement, the slides were reviewed again and a consensus was reached. To select the cutoff value, we calculated the $P$-value in log-rank (LR) survival analysis at each score of HSF1 and MCT4 expression in tumour and matched peritumoral tissue. A different $P$-value at each score was present in line chart and we can easily find which immunomarker has a long range of cutoff values for $P<0.05$ or $P<0.01$. To investigate the relationship between HSF1 and MCT4 in peritumoral tissue, the MCT4 was measured using a computerised image system composed of Leica CCD camera DFC420 connected to a Leica DM IRE2 microscope (Leica Microsystems Imaging Solutions, Cambridge, UK) (Zhu et al, 2008).

Cell culture and hypoxia. Human hepatic cell line L02, low metastatic potential human HCC cell line PLC (obtained from the Cell Bank of the Chinese Academy of Sciences, Shanghai, China) and MHCC-97H cells (human HCC cell lines with high metastatic potential, established at the Liver Cancer Institute, Zhongshan Hospital, Fudan University, Shanghai, China) were maintained in Dulbecco's modified Eagle's medium containing $10 \%$ foetal calf serum, $100 \mathrm{U} \mathrm{ml}^{-1}$ penicillin and $50 \mu \mathrm{g} \mathrm{ml}^{-1}$ streptomycin. Hypoxic cells were incubated at $37^{\circ} \mathrm{C}$ under a humidified atmosphere $\left(5 \% \mathrm{CO}_{2}\right)$ for $8 \mathrm{~h}$ in a hypoxia incubator chamber (the Ruskinn Invivo 2 200; Ruskinn Technologies, Leeds, UK) with $1 \% \mathrm{O}_{2}$ and $5 \% \mathrm{CO}_{2}$ (90\% humidity). The cells were then used for follow-up experiments. 
Western blot. Equivalent protein amounts were denatured in an SDS sample buffer. Then, samples were separated on a denaturing $10 \%$ SDS-polyacrylamide gel. Proteins were transferred onto polyvinylidene difluoride membrane and were blocked with $5 \%$ non-fat dry milk in PBS containing $0.05 \%$ Tween-20. Membranes were incubated with primary antibodies that recognise HSF1 ( $1: 2000$; Cell Signaling Technology), MCT4 (1:1000, Santa Cruz Biotechnology). Western blot analysis was carried out using the enhanced chemiluminescence detection system (Perkin-Elmer Life Sciences, Rockford, IL, USA) and developed on a film. Quantification of western blots was analysed densitometrically using Quantity One software (Bio-Rad Laboratories, Inc., Hercules, CA, USA).

Construction of LV-HSF1-shRNA vector and transfection. Lentiviral vectors pLKO.1 TRC and pWPI.1 were used for constructing recombinant lentiviruses. Oligonucleotides encoding hairpin precursors for shHSF1 (target sequence: 5'-CCGGCAGGAGCA GCTCCTTGAGATTCAAGAGATCTCAAGGAGCTGCTCCTGT TTTTG- $3^{\prime}$ ) were used for generating short interference RNA (shRNA) constructs. A scrambled non-targeting sequence was used as a control (non-targeting shRNA (shNT)). Synonymous mutations were introduced into the target sequence of shHSF1 (5'-CAGGAACAGCTTCTCGAGA-3', mutations underlined) in HSF1 ORF to generate shHSF1-resistant HSF1(shRES). Recombinant lentivirus was amplified in HEK293T cells.

In vitro proliferation assays. MHCC-97H and PLC cells were seeded at 3000 cells per well in 96-well plates and were cultivated in the supernatant of shNT- or shHSF1-treated L02 cells in normoxia and hypoxia conditions. The CCK8 assay was used to determine the relative viability of cells according to the manufacturer's instructions. This procedure was repeated at indicated times when the cells were cultivated in the corresponding supernatant.

Colony formation assay. Hepatocellular carcinoma cells cultured with the supernatant of shNT- or shHSF1-treated L02 cells in normoxia and hypoxia conditions for $48 \mathrm{~h}$ were trypsinised and counted. Cells were then placed in 5000 cells per $750 \mu \mathrm{l} \mathrm{L02}$ supernatant and mixed with $250 \mu \mathrm{l} 1.2 \%$ agar. One millilitre mixture was added onto base agar in each $35 \mathrm{~mm}$ dish. Dishes were incubated at $37^{\circ} \mathrm{C}$ in humidified incubator for 2 weeks to count colonies. Each assay was performed in triplicate on two independent occasions.

Statistical analysis. Analysis was performed with SPSS 13.0 for windows (SPSS Inc., Chicago, IL, USA). The Pearson's $\chi^{2}$ test or Fisher's exact test was used to compare qualitative variables, and quantitative variables were expressed as the means \pm s.d. and were analysed by the $t$-test or Pearson's correlation test. Survival curves were computed with Kaplan-Meier analysis and were compared between subgroups through the LR test. The Cox regression model was used to perform multivariate analysis. Receiver operating characteristic curve analysis was used to determine the predictive value of the parameters. $P<0.05$ was considered statistically significant.

\section{RESULTS}

Patients' general characteristics and OS and TTR. On 31 March 2010, the median survival of 105 patients in cohort 1 was 65.0 months and approximately $50 \%$ of patients were alive at 5 years, similar to previous 5-year survival figures reported for patients after radical hepatectomy (Chok et al, 2009). For the recurrent patients $(n=63)$, treatment modalities including resection $(n=18)$, radiofrequency ablation $(n=3)$, transcatheter arterial chemoembolisation $(n=35)$ and radiotherapy $(n=2)$ were administered according to a uniform guideline, with 10 patients receiving support treatment only because of poor liver function. Most patients in our series had hepatitis B background, with $25.7 \%$ of $\mathrm{HBeAg}$ positive and $78.1 \%$ of liver cirrhosis. The cumulative
$1-, 3$ - and 5-year OS rates were, respectively $83 \%, 63 \%$ and $50 \%$. The 1-, 3- and 5-year probabilities of recurrence were, respectively, $27 \%, 51 \%$ and $66 \%$.

HSF1 expression in HCC and surrounding non-tumoral liver tissues and their potential cutoff values. Heat-shock transcription factor-1 immunostaining was mainly confined to the nucleus with tumour cells and peritumoral hepatocytes showing moderateto-intense positivity, as opposed to relatively weaker expression in the extracellular matrix and most other local inflammatory cells excluding the bile duct cells (Figure 1A). Cores of two patients for peritumoral HSF1 immunostaining were unexpectedly detached from TMA without sufficient tissue to score. Minimum $P$-value approach (Galon et al, 2006) for HSF1 was used, and $P$-value was calculated in LR survival analysis using each staining score of intratumoral and peritumoral HSF1 as a cutoff value. Statistical significance was observed for more than $50 \%$ peritumoral HSF1 staining scores (Supplementary Figures S3 and S4). Lastly, the cutoff value was set at the median score as usual for HSF1, although the $P$-value is not the best at this score.

Peritumoral HSF1 expression associated with prognosis in univariate and multivariate analysis. Using Kaplan-Meier analysis patients with high peritumoral HSF1 expression had a significantly worse prognosis than HCC patients with low peritumoral HSF1 expression in cohort $1(\mathrm{LR}=13.378, P<0.001$; Figure 1B). The median survival was 38 months (95\% CI: 22-54 months) and there were 33 deaths in 48 patients with high peritumoral HSF1 compared with median survival of 115 months (95\% CI: 56-174 months) and 20 deaths in 55 patients with low peritumoral HSF1 expression. In addition, peritumoral HSF1 was associated with TTR ( $\mathrm{LR}=14.45, P<0.001$; Figure $1 \mathrm{~B})$. The mean TTR in patients with high peritumoral HSF1 expression was 13 months (95\% CI: 7-19 months), whereas it was up to 59 months (95\% CI: 46-72 months) in patients with low peritumoral HSF1 expression (Figure 1B). However, HSF1 expression in intratumoral tissues was not significantly associated with OS $(P=0.067)$ or TTR $(P=0.302)$. To further confirm such association between peritumoral HSF1 expression and HCC patients' prognosis, we investigated HSF1 expression in peritumoral liver tissue from 227 HCC patients in validation cohort. Using the same cutoff value of peritumoral HSF1 expression in cohort 1, the statistical results showed peritumoral HSF1 expression, in accordance with the results in cohort 1 , can predict patients' OS $(P<0.001)$ and TTR $(P<0.001)$. The clinicopathologic features of patients from two cohorts enrolled in our study were listed in Supplementary Table S2.

As recurrence occurs in $70-100 \%$ of cases following HCC resection surgery, and early detection of recurrence is clinically important for improving prognosis, we conducted a further study to demonstrate the value of peritumoral HSF1 on predicting ER. Using 2 years as cutoff value (Poon et al, 2000), we found that patients with high peritumoral HSF1 expression tended to have an ER $(P<0.001)$ rather than late recurrence $(P=0.576)$. However, intratumoral HSF1 expression was not associated with early $(P=0.771)$ or late recurrence $(P=0.535)$ (Figure 2$)$.

In addition, multivariate Cox proportional hazards analysis including factors that showed significant predictive values in OS and TTR in univariate analysis was performed (Table 1). The result indicated that peritumoral HSF1 was still an independent risk factor for both OS (hazard ratio (Oehme et al, 2002) $=2.60$, $P=0.002)$ and TTR $(\mathrm{HR}=2.52, P<0.001)$.

High peritumoral HSF1 correlations with clinicopathological factors and HSF1's predictive value in subgroups. As shown in Supplementary Table S3, there was no correlation between intratumoral HSF1 and any clinicopathologic feature, whereas patients with high peritumoral HSF1 level were prone to larger tumour size, higher AFP levels and ER. Univariate analysis showed 
A
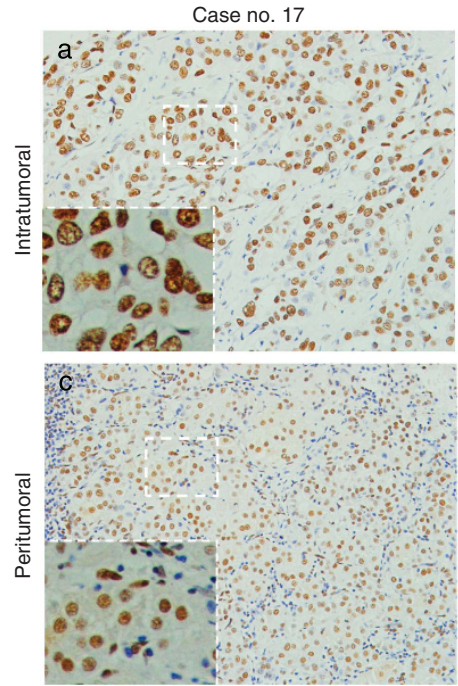

B

B

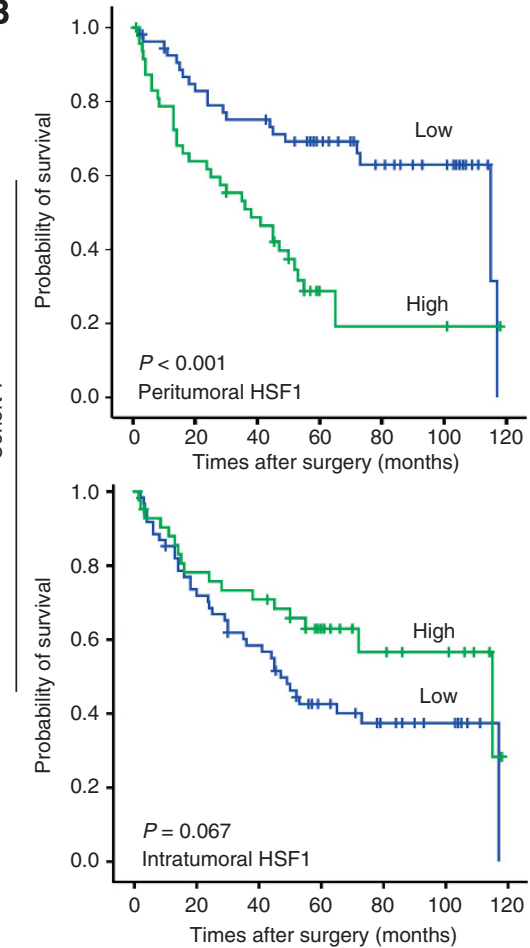

C

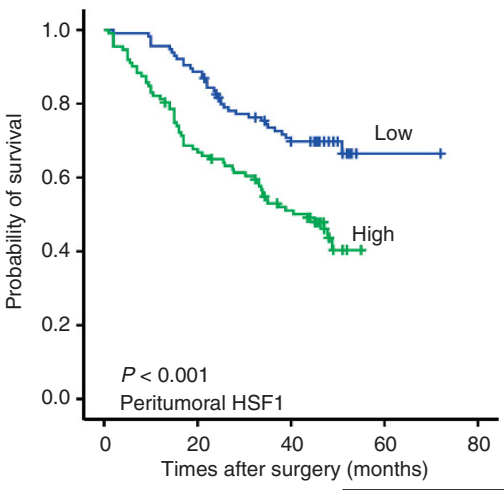

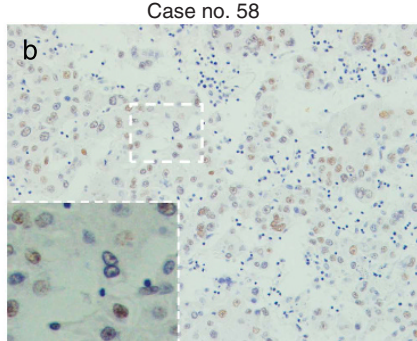
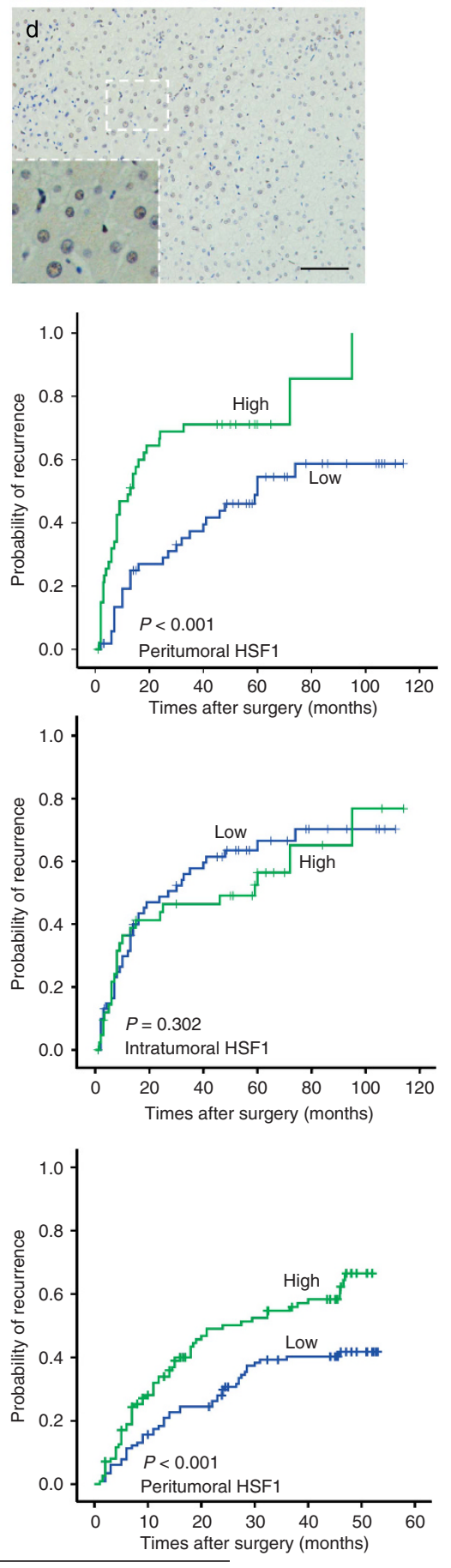

Validation cohort

Figure 1. Heat-shock transcription factor-1 is expressed in HCC and matched peritumoral liver tissue and its expression in peritumoral liver tissue correlates with clinical outcomes of patients with HCC. (A) Hepatocellular carcinoma representative strong (case no. 17) and mild (case no. 58) immunostaining are shown for HSF1 in intratumoral $(a, b)$ and peritumoral (c, d) tissue (scale bar, $100 \mu$ m). (B) Ten-year Kaplan-Meier survival plots of HSF1 for OS and TTR in peritumoral liver tissue and HCC tissue in cohort 1, and (C) peritumoral HSF1 for OS and TTR in validation cohort. Log-rank $P$-values associated with each dichotomisation are shown in the lower left of each plot. 

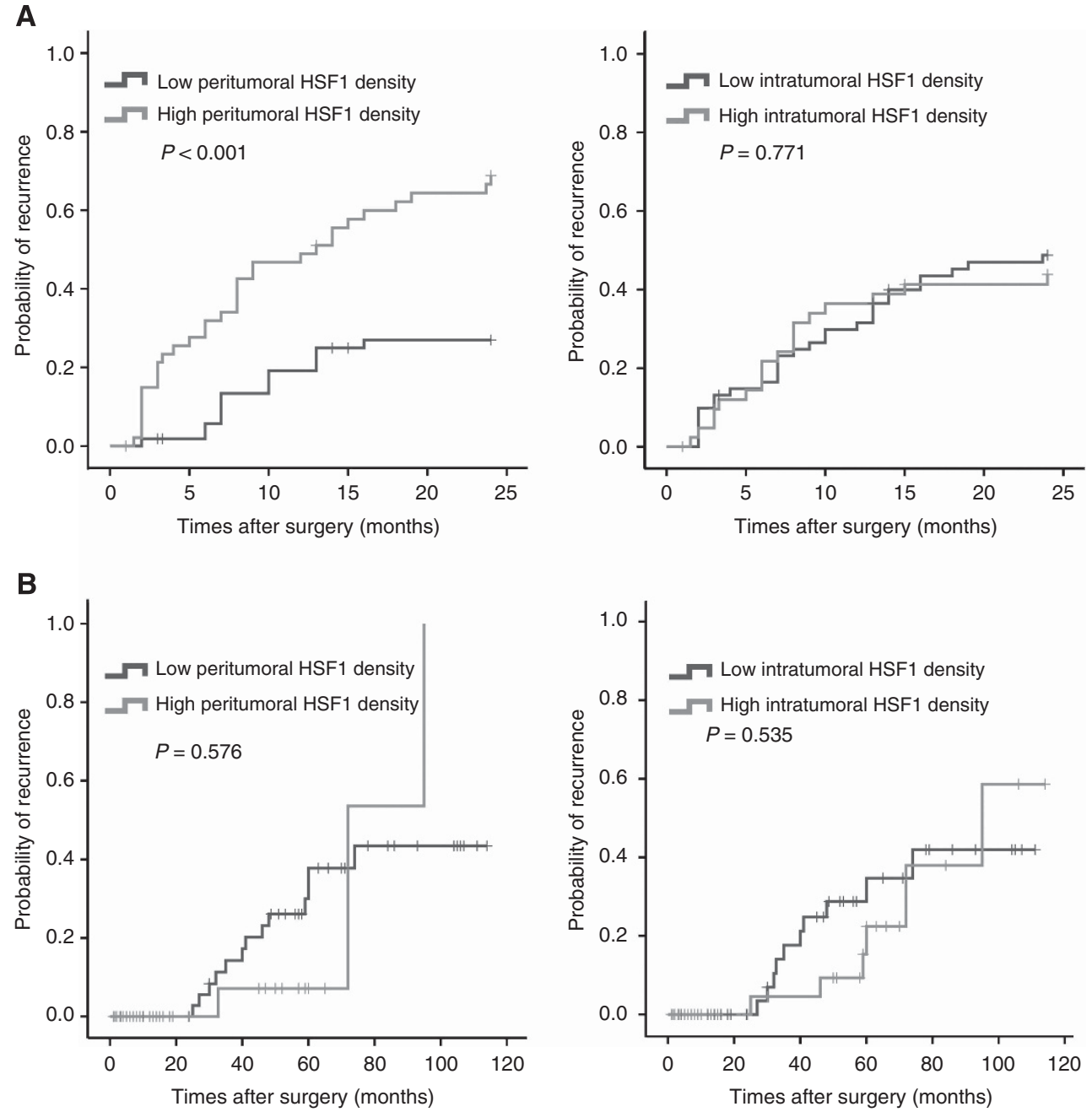

Figure 2. The predictive role of HSF1 expression in peritumoral liver tissue for ER. (A) Using 2 years as a cutoff value, postoperative recurrence was discriminated into early and later recurrence according to the TTR. Early recurrence curves, but not late recurrence curves, differed between high and low peritumoral HSF1 expression. (B) Early or late recurrence did not differ between patients with high and low intratumoral HSF1 expression.

that larger tumour size and higher AFP levels were significantly associated with worse prognosis of HCC patients. Therefore, we attempted to reduce the impact of tumour size and serum AFP level on patients' survival during the evaluation of HSF1's predictive power on OS and TTR. We assessed the predictive value of peritumoral HSF1 in small tumour size (the diameter of tumour $\leqslant 5 \mathrm{~cm}, n=56$ ) and low AFP (AFP $\leqslant 400 \mathrm{ng} \mathrm{ml}^{-1}$, $n=64$ ) subgroups showing that the level of peritumoral HSF1 was significantly associated with OS and TTR in these subgroups (Supplementary Figure S5). The further Cox regression analysis showed that among small-HCC subgroup, the high peritumoral HSF1 level predicted poor OS $(\mathrm{HR}=6.21, P=0.001)$ and TTR $(\mathrm{HR}=2.23, P=0.03)$. The similar results were also found in the low AFP subgroup (for OS: $\mathrm{HR}=2.64, P=0.022$; for TTR: $\mathrm{HR}=2.06, P=0.036)$ (Supplementary Figure S6).

Effects of HSF1 alteration in peritumoral liver cells on proliferation and colonisation of HCC cells. It is well known that hypoxia and oxidative stress occur in peritumoral liver microenvironment and have an important influence on the progression of HCC. To clarify the effects of high HSF1 expression in peritumoral liver tissue on HCC cells, the L02 cell was treated with shNT or individual shRNA against HSF1 (shHSF1 nos. 1-3; Supplementary Figure S7). shHSF1 no. 3, which induces the most significant knockdown effects (about $80 \%$ reduction on HSF1 expression), was adopted for further study. The supernatants from L02 cells with efficient shHSF1 treatment in normoxia or hypoxia condition were added into the medium of MHCC-97H. Suppression of colonisation (Figure 3A) and proliferation (Figure 3B) was found when MHCC-97H was cultured with the supernatant of shHSF1-treated L02 in hypoxia as compared with shNT-treated L02 in hypoxia. There is no difference when HCC cells cultured with supernatant of shHSF1- and shNT-treated L02 in normoxia. Similar inhibition effects occurred in PLC with the same treatment (data not shown). Notably, the specificity of shHSF1-mediated effects was further documented by proliferation rescue of L02 by an HSF1 complementary DNA engineered to be insensitive to shRNA (Figure 3C). Taken together, these genetic loss-of-function studies using RNA interference-mediated knockdown indicated that high expression of HSF1 in peritumoral liver cells is critical for HCC proliferation and colonisation.

The prognostic value of combining peritumoral HSF1 and MCT4 and ROC analysis. In normoxia and hypoxia culture condition, HSF1 deficiency in L02 cells significantly downregulated MCT4 expression. Furthermore, MCT4 expression increased when shHSF1-L02 cells were transfected with HSF1-cDNA engineered to be insensitive to shRNA (Figure 3D). On the basis of the in vitro finding that HSF1 regulated MCT4 expression in liver cell, we further investigated the relationship in peritumoral liver tissues. 


\section{Table 1. Univariate and multivariate analysis of factors associated with survival and recurrence}

\begin{tabular}{|c|c|c|c|c|c|c|c|c|}
\hline \multirow[b]{3}{*}{ Features } & \multicolumn{4}{|c|}{ Overall survival } & \multicolumn{4}{|c|}{ Time to recurrence } \\
\hline & \multirow{2}{*}{$\begin{array}{c}\text { Univariate } \\
P \text {-value }\end{array}$} & \multicolumn{3}{|c|}{ Multivariate } & \multirow{2}{*}{$\begin{array}{c}\text { Univariate } \\
\boldsymbol{P} \text {-value }\end{array}$} & \multicolumn{3}{|c|}{ Multivariate } \\
\hline & & Hazard ratio & $95 \% \mathrm{Cl}$ & $\boldsymbol{P}$-value & & Hazard ratio & $95 \% \mathrm{Cl}$ & $P$-value \\
\hline Age: $<51$ vs $\geqslant 51$ years & 0.945 & & & NA & 0.589 & & & NA \\
\hline Gender: female vs male & 0.186 & & & NA & 0.078 & & & NA \\
\hline Hepatitis B history: yes vs no & 0.946 & & & NA & 0.807 & & & NA \\
\hline HBeAg: positive vs negative & 0.338 & & & NA & 0.293 & & & NA \\
\hline Liver cirrhosis: yes vs no & 0.006 & & & NS & 0.097 & & & NA \\
\hline AFP: $<400$ vs $\geqslant 400 \mathrm{ng} \mathrm{ml}^{-1}$ & 0.003 & & & NS & 0.052 & & & NA \\
\hline Preoperative ALT: $\leqslant 75$ vs $>75 \mathrm{UI}^{-1}$ & 0.668 & & & NA & 0.409 & & & NA \\
\hline Tumour size: $\leqslant 5$ vs $>5 \mathrm{~cm}$ & $<0.001$ & 1.90 & $1.03-3.53$ & 0.042 & $<0.001$ & & & NS \\
\hline Encapsulation: complete vs none & 0.077 & & & NA & 0.086 & & & NA \\
\hline Microvascular invasion: yes vs no & 0.017 & & & NS & 0.105 & & & NA \\
\hline Intrahepatic metastasis: yes vs no & $<0.001$ & & & NS & 0.001 & & & NS \\
\hline TNM stage: IIla vs II vs I & $<0.001$ & 1.99 & $1.40-2.83$ & $<0.001$ & $<0.001$ & 2.04 & $1.47-2.82$ & $<0.001$ \\
\hline Intratumoral HSF1 level: low vs high & 0.067 & & & NA & 0.302 & & & NA \\
\hline Peritumoral HSF1 level: low vs high & $<0.001$ & 2.60 & $1.44-4.70$ & 0.002 & $<0.001$ & 2.52 & $1.51-4.19$ & $<0.001$ \\
\hline
\end{tabular}

It was found that MCT4 expression displayed a diffuse plasma membrane and/or cytoplasmic pattern including liver cells and endothelial cells (Supplementary Figure S8) and peritumoral MCT4 expression was correlated with the peritumoral HSF1 expression significantly $(P=0.014$, Figure $4 \mathrm{~A})$. Moreover, patients with high peritumoral MCT4 level had poor OS $(P=0.003)$ and TTR $(P=0.007$; Supplementary Figure $S 9)$. To further improve the prognostic value, peritumoral HSF1 and MCT4 were combined and patients were classified into four groups according to median value of their HSF1 and MCT4 expression (Figure 4B): group I $(n=27)$, low HSF1 and low MCT4 (HSF1 $\downarrow$ MCT4 $\downarrow)$; group II $(n=28)$, low HSF1 but high MCT4 (HSF1 $\downarrow$ MCT4 $\uparrow$ ); group III $(n=14)$, high HSF1 but low MCT4 (HSF1 $\uparrow$ MCT4 $\downarrow$ ); and group IV $(n=34)$, high HSF1 and high MCT4 (HSF1 $\uparrow$ MCT4 $\uparrow)$. There were significant differences in both OS $(P<0.001)$ and TTR $(P<0.001)$ among the four groups. Notably, 5-year OS and TTR rates were $76 \%$ and $57 \%$, respectively, in group I and $30 \%$ and $25 \%$, respectively, in group IV.

Clinicopathologic factors showing significance in multivariate survival analysis and the combination of peritumoral HSF1 and MCT4 were included in ROC analysis. The result showed that HSF1 alone could predict death and recurrence precisely with the area under the curve, $0.661(P=0.005)$ and $0.649(P=0.009)$, respectively. The combination of HSF1 and MCT4 further elevated the area under the curve, and was better for predicting death $(0.707,95 \% \mathrm{CI}=0.606-0.808)$ and recurrence $(0.676$, $95 \% \mathrm{CI}=0.572-0.780)$. For the $\mathrm{ER}$, the area under the curve was up to $0.759(95 \% \mathrm{CI}=0.665-0.853)$ (Figure $4 \mathrm{C}$ ). The specific values of all predicted factors were seen in Supplementary Table S4.

\section{DISCUSSION}

Peritumoral liver microenvironment factors, especially those representing the multifaceted situation objectively, had a key impact on HCC progression (Hoshida et al, 2008), and may be potential intervention targets to further improve prognosis. It is well known that a wide variety of environmental stresses can induce HSPs expression, which conversely mediates resistance to further stress (Nahleh et al, 2012). High expression of HSPs has been demonstrated in some cancer tissues and is correlated clinically with the severity of tumours and poor outcomes. For example, other reports (Yang et al, 2010) and our previous studies (Feng et al, 2005; Guo et al, 2008) showed that HSP27 was a prognostic marker associated with HCC cell motility and invasion. Other HSPs such as HSP70 and HSP90 also reported overexpression in HCC through chaperoning many signal transducers (Wang, 2011; Neckers and Workman, 2012). To our knowledge, expression of HSPs depends ultimately on transcription factorHSF1 activation, which causes rapid transcription of HSP genes to permit survival of cells and restoration of global protein quality (Neckers and Workman, 2012).

Heat-shock transcription factor- 1 has been implicated in the pathogenesis of cancer, but its clinical significance remains elusive. Here, based on the analysis of two HCC patients cohorts, we reported two key insights for the first time: (1) HCC patients with high expression of peritumoral HSF1, but not intratumoral HSF1, have a higher incidence of ER and poor OS, even in patients with slight HCC or low AFP level; (2) the power of peritumoral HSF1 in predicting HCC patients' prognosis was independent of other significant clinicopathological factors. Furthermore, using the Scheuer score system - the semiquantitative staging of inflammation and fibrosis in chronic hepatitis (Stuwe et al, 2007; Wang, 2011), we evaluated the peritumoral intrahepatic inflammation status and found no significant correlation between peritumoral HSF1 levels and the inflammation score in liver tissues. This suggests that besides inflammatory stress, some other stresses may also be involved in regulating HSF1 level such as hypoxia, abnormal metabolism and so on. Intriguingly, in our in vitro study, the supernatant of shHSF1-treated L02 in hypoxia, NOT normoxia condition, inhibited colonisation and proliferation of HCC cells. This indicated that compared with physiological conditions, a microenvironment orchestrated by HSF1 under stressful conditions is more likely to affect HCC cells.

Although HSF1 is not an oncogene, increasing evidence has demonstrated that it is a powerful multifaceted modifier of 
A

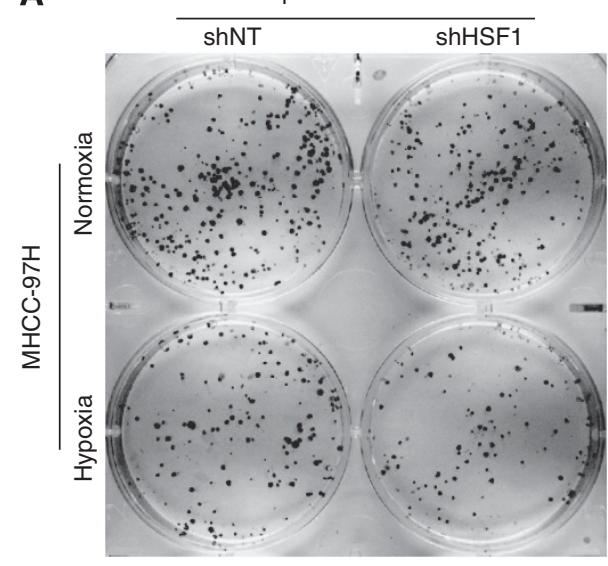

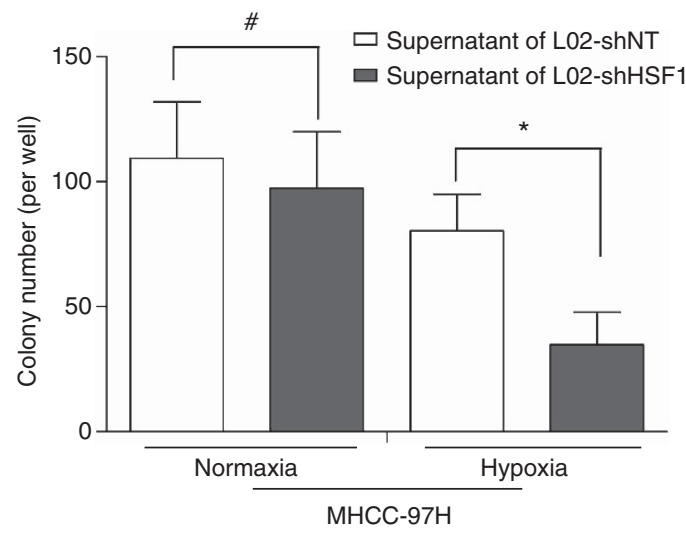

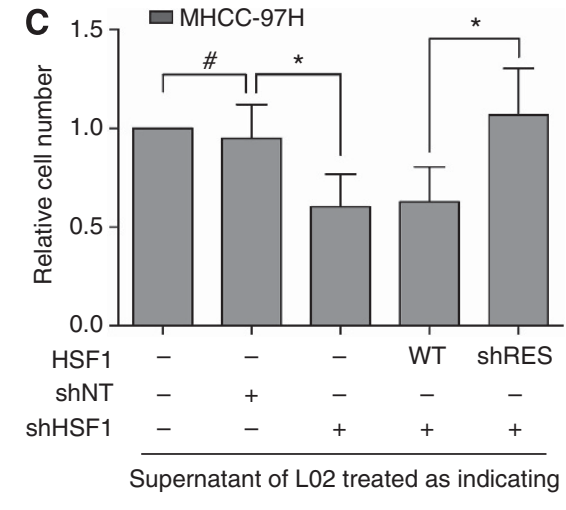

Supernatant of L02 treated as indicating

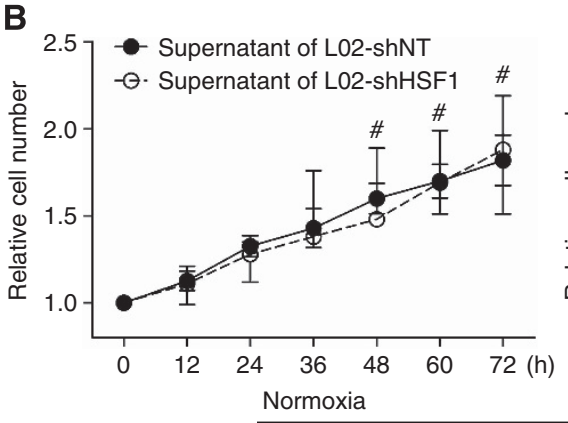

MHCC-97H

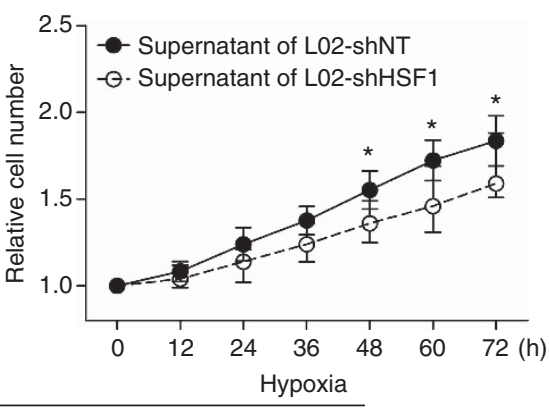

shHSF

D
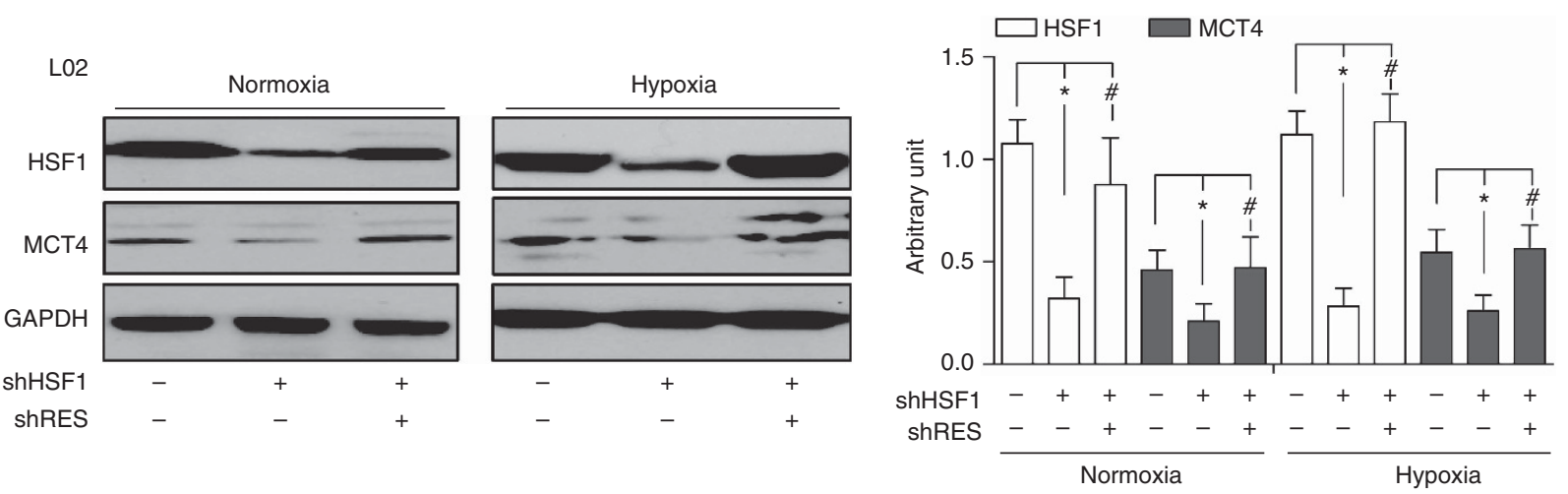

Figure 3. Effects of HSF1 alteration in peritumoral liver cells on proliferation and colonlisation of HCC cells, and the expression of MCT4.

(A) Compared with that of L02-shNT cells, the supernatant of L02-shHSF1 cells in hypoxia condition significantly suppressed MHCC-97H colony formation and proliferation. (B) Using the supernatant in normoxia condition, there was no difference between colony formation and proliferation between two groups. (C) The specificity of shHSF1-mediated effects was further documented by reintroducing HSF1-cDNA engineered to be insensitive to shRNA. (D) In both normoxia and hypoxia culture conditions, silencing the expression of HSF1 led to low MCT4 expression.

carcinogenesis. Lindquist (Dai et al, 2007) reported that the effects of HSF1 on modulating initiation and progression are far beyond HSPs induction. Cumulative observations suggested that HSF1 is linked to an altered metabolism in cancer. As a major transactivator of stress proteins, HSF1 activation stimulated liver cell lipid biosynthesis and perpetuated chronic hepatic metabolic disease induced by carcinogens through the promotion of premalignant cell growth in a mouse model (Jin et al, 2011). In addition, HSF1 can modulate glucose metabolism by increasing the rate of glycolysis (Dai et al, 2007). These studies implied that HSF1 has a central role in HCC development, as it modulates proteostasis and metabolic pathways by regulating access to two critical elements: glucose and lipids. The metabolic shift from oxidative phosphorylation to glycolysis contributes to the acidic microenvironment. To survive through the acidic microenvironment, cells need MCTs to maintain the acid-resistant status (Dhup et al, 2012). Among these MCTs, MCT4 has a key role by exporting newly formed lactate, and allowing continuous conversion of pyruvate to lactate in cells with high glycolytic rates related to hypoxic energy production (Pinheiro et al, 2012).

In this study, it was observed that MCT4 was downregulated when L02 was transfected with shHSF1, and was upregualted almost back to original level when shHSF1 L02 cells were transfected with HSF1-cDNA engineered to be insenstitive to shRNA. This suggested that MCT4 is a target gene of HSF1 in liver cells. In addition, we also found in cohort 1 that peritumoral MCT4 expression was increased significantly in high peritumoral HSF1. The results were similar to reports that the HSF1-dependent stress response has evolved to enhance survival (Zhao et al, 2009) in the face of environmental challenges such as hypoxia and chronic 

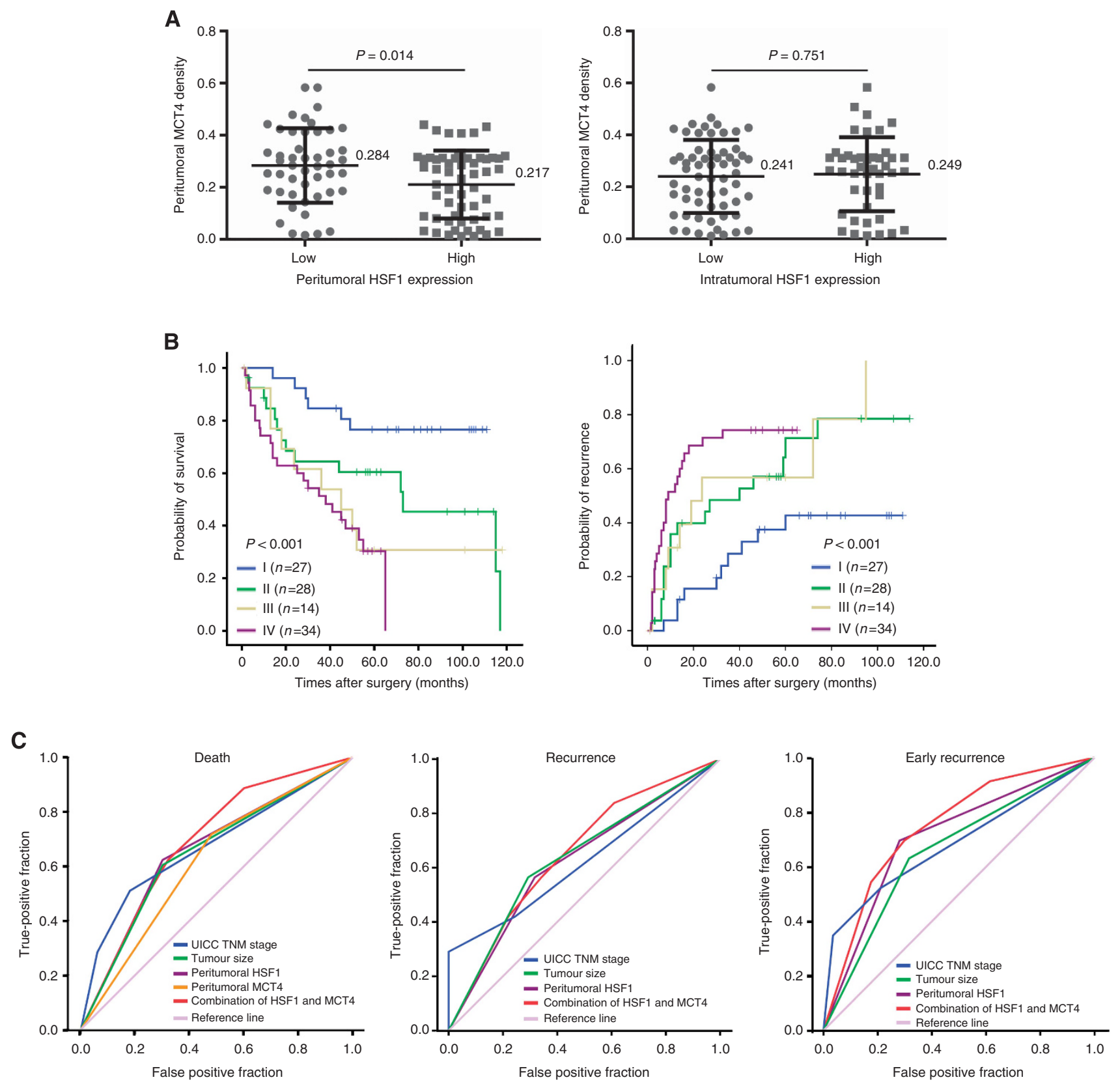

Figure 4. Prognostic and predictive value of combining peritumoral HSF1 and MCT4. (A) The expression of peritumoral HSF1 was associated with the expression of peritumoral MCT4 expression, whereas there is no correlation between intratumoral HSF1 and peritumoral MCT4 expression. (B) The cumulative OS and TTR curves of the combination of pertitumoral HSF1 and MCT4. (C) All factors adopted in ROC analysis predicted death (left panel), recurrence (middle panel) and ER (right panel) during follow-up. The predictive value of HSF1 combined with MCT4 was the best in predicting ER.

inflammation (Hahn et al, 2004; Ando et al, 2010). Furthermore, the combination of peritumoral HSF1 and MCT4 expression could improve the predictive level for OS, especially for ER, given that the area under the curve of ER is the best among all factors.

In summary, our results suggested that high peritumoral HSF1 expression can serve as a sensitive 'readout' for the high-risk early recurrent HCC patient receiving radical resection. In consideration of HSF1's pivotal role in the cellular response to stress, particularly the sensitive response of cirrhotic liver cell metabolism to inflammatory stimulation, HSF1, which helps cancer cells and their outside environment communicate with one another, could have more superiorities than other single factors in reflecting the cells status in general. So, HSF1 could be a potential intervention target for comprehensive therapy of HCC and deserves further investigation.

\section{ACKNOWLEDGEMENTS}

We thank Mr Wei-De Zhang for assistance in collecting patient data. This study was jointly supported by three grants (Nos. 30872505, 81001057 and 81372654) from the National Natural Science Foundation of China, and the Research Fund for the Doctoral Program of Higher Education of China (No. 200802461037).

\section{CONFLICT OF INTEREST}

The authors declare no conflict of interest. 


\section{REFERENCES}

Alison MR, Nicholson LJ, Lin WR (2011) Chronic inflammation and hepatocellular carcinoma. Recent results. Cancer Res 185: 135-148.

Ando M, Uehara I, Kogure K, Asano Y, Nakajima W, Abe Y, Kawauchi K, Tanaka N (2010) Interleukin 6 enhances glycolysis through expression of the glycolytic enzymes hexokinase 2 and 6-phosphofructo-2-kinase/ fructose-2,6-bisphosphatase-3. J Nihon Med Sch 77: 97-105.

Brahimi-Horn MC, Bellot G, Pouyssegur J (2011) Hypoxia and energetic tumour metabolism. Curr Opin Genet Dev 21: 67-72.

Brahimi-Horn MC, Pouyssegur J (2007) Hypoxia in cancer cell metabolism and $\mathrm{pH}$ regulation. Essays Biochem 43: 165-178.

Bremnes RM, Veve R, Gabrielson E, Hirsch FR, Baron A, Bemis L, Gemmill RM, Drabkin HA, Franklin WA (2002) High-throughput tissue microarray analysis used to evaluate biology and prognostic significance of the E-cadherin pathway in non-small-cell lung cancer. J Clin Oncol 20(10): 2417-2428.

Brunt EM (2000) Grading and staging the histopathological lesions of chronic hepatitis: the Knodell histology activity index and beyond. Hepatology 31(1): 241-246.

Calderwood SK, Khaleque MA, Sawyer DB, Ciocca DR (2006) Heat shock proteins in cancer: chaperones of tumorigenesis. Trends Biochem Sci 31: 164-172.

Chok KS, Ng KK, Poon R, Lo CM, Fan ST (2009) Impact of postoperative complications on long-term outcome of curative resection for hepatocellular carcinoma. Br J Surg 96: 81-87.

Dai C, Whitesell L, Rogers AB, Lindquist S (2007) Heat shock factor 1 is a powerful multifaceted modifier of carcinogenesis. Cell 130: 1005-1018.

Dhup S, Dadhich RK, Porporato PE, Sonveaux P (2012) Multiple biological activities of lactic acid in cancer: influences on tumor growth, angiogenesis and metastasis. Curr Pharm Des 18: 1319-1330.

Feng JT, Liu YK, Song HY, Dai Z, Qin LX, Almofti MR, Fang CY, Lu HJ, Yang PY, Tang ZY (2005) Heat-shock protein 27: a potential biomarker for hepatocellular carcinoma identified by serum proteome analysis. Proteomics 5: 4581-4588.

Ferlay JSH, Bray F, Forman D (2010) GLOBOCAN 2008, Cancer Incidence and Mortality Worldwide: IARC Cancer Base No. 10 (Internet). International Agency for Research on Cancer: Lyon, France. Available at http://globocaniarcfr.

Galon J, Costes A, Sanchez-Cabo F, Kirilovsky A, Mlecnik B, Lagorce-Pages C, Tosolini M, Camus M, Berger A, Wind P (2006) Type, density, and location of immune cells within human colorectal tumors predict clinical outcome. Science 313: 1960-1964.

Guo K, Liu Y, Zhou H, Dai Z, Zhang J, Sun R, Chen J, Sun Q, Lu W, Kang X, Chen P (2008) Involvement of protein kinase $C$ beta-extracellular signalregulating kinase $1 / 2 / \mathrm{p} 38$ mitogen-activated protein kinase-heat shock protein 27 activation in hepatocellular carcinoma cell motility and invasion. Cancer Sci 99: 486-496.

Hahn JS, Hu Z, Thiele DJ, Iyer VR (2004) Genome-wide analysis of the biology of stress responses through heat shock transcription factor. Mol Cell Biol 24: 5249-5256.

Halestrap AP, Wilson MC (2012) The monocarboxylate transporter family - role and regulation. IUBMB Life 64: 109-119.

Hoshida Y, Villanueva A, Kobayashi M, Peix J, Chiang DY, Camargo A, Gupta S, Moore J, Wrobel MJ, Lerner J (2008) Gene expression in fixed tissues and outcome in hepatocellular carcinoma. N Engl J Med 359: 1995-2004.

Huber V, De Milito A, Harguindey S, Reshkin SJ, Wahl ML, Rauch C, Chiesi A, Pouyssegur J, Gatenby RA, Rivoltini L, Fais S (2010) Proton dynamics in cancer. J Transl Med 8: 57.

Jin X, Moskophidis D, Mivechi NF (2011) Heat shock transcription factor 1 is a key determinant of HCC development by regulating hepatic steatosis and metabolic syndrome. Cell Metab 14: 91-103.

Luk JM, Lam CT, Siu AF, Lam BY, Ng IO, Hu MY, Che CM, Fan ST (2006) Proteomic profiling of hepatocellular carcinoma in Chinese cohort reveals heat-shock proteins (Hsp27, Hsp70, GRP78) up-regulation and their associated prognostic values. Proteomics 6: 1049-1057.

Nahleh Z, Tfayli A, Najm A, El Sayed A, Nahle Z (2012) Heat shock proteins in cancer: targeting the 'chaperones'. Fut Med Chem 4: 927-935.

Neckers L, Workman P (2012) Hsp90 molecular chaperone inhibitors: are we there yet? Clin Cancer Res 18: 64-76.

Oehme F, Ellinghaus P, Kolkhof P, Smith TJ, Ramakrishnan S, Hutter J, Schramm M, Flamme I (2002) Overexpression of PH-4, a novel putative proline 4-hydroxylase, modulates activity of hypoxia-inducible transcription factors. Biochem Biophys Res Commun 296: 343-349.

Palazon A, Aragones J, Morales-Kastresana A, de Landazuri MO, Melero I (2012) Molecular pathways: hypoxia response in immune cells fighting or promoting cancer. Clin Cancer Res 18: 1207-1213.

Pertega-Gomes N, Vizcaino JR, Miranda-Goncalves V, Pinheiro C, Silva J, Pereira H, Monteiro P, Henrique RM, Reis RM, Lopes C, Baltazar F (2011) Monocarboxylate transporter 4 (MCT4) and CD147 overexpression is associated with poor prognosis in prostate cancer. $B M C$ Cancer 11: 312.

Pinheiro C, Longatto-Filho A, Azevedo-Silva J, Casal M, Schmitt FC, Baltazar F (2012) Role of monocarboxylate transporters in human cancers: state of the art. J Bioenerg Biomembr 44: 127-139.

Poon RT, Fan ST, Ng IO, Lo CM, Liu CL, Wong J (2000) Different risk factors and prognosis for early and late intrahepatic recurrence after resection of hepatocellular carcinoma. Cancer 89: 500-507.

Severi T, van Malenstein H, Verslype C, van Pelt JF (2010) Tumor initiation and progression in hepatocellular carcinoma: risk factors, classification, and therapeutic targets. Acta Pharmacol Sin 31: 1409-1420.

Sherman M, Multhoff G (2007) Heat shock proteins in cancer. Ann N Y Acad Sci 1113: 192-201.

Stuwe L, Muller M, Fabian A, Waning J, Mally S, Noel J, Schwab A, Stock C (2007) $\mathrm{pH}$ dependence of melanoma cell migration: protons extruded by NHE1 dominate protons of the bulk solution. J Physiol 585: 351-360.

Tang ZY, Ye SL, Liu YK, Qin LX, Sun HC, Ye QH, Wang L, Zhou J, Qiu SJ, Li Y (2004) A decade's studies on metastasis of hepatocellular carcinoma. J Cancer Res Clin Oncol 130: 187-196.

Thorgeirsson SS, Grisham JW (2002) Molecular pathogenesis of human hepatocellular carcinoma. Nat Genet 31: 339-346.

Tinkle CL, Haas-Kogan D (2012) Hepatocellular carcinoma: natural history, current management, and emerging tools. Biologics 6: 207-219.

Vander Heiden MG (2011) Targeting cancer metabolism: a therapeutic window opens. Nat Rev Drug Discov 10: 671-684.

Wang RE (2011) Targeting heat shock proteins 70/90 and proteasome for cancer therapy. Curr Med Chem 18: 4250-4264.

Whitesell L, Lindquist S (2009) Inhibiting the transcription factor HSF1 as an anticancer strategy. Expert Opin Ther Targets 13: 469-478.

Yang F, Yin Y, Wang F, Wang Y, Zhang L, Tang Y, Sun S (2010) miR-17-5p Promotes migration of human hepatocellular carcinoma cells through the p38 mitogen-activated protein kinase-heat shock protein 27 pathway. Hepatology 51: 1614-1623.

Zhao YH, Zhou M, Liu H, Ding Y, Khong HT, Yu D, Fodstad O, Tan M (2009) Upregulation of lactate dehydrogenase A by ErbB2 through heat shock factor 1 promotes breast cancer cell glycolysis and growth. Oncogene 28: 3689-3701.

Zhu XD, Zhang JB, Zhuang PY, Zhu HG, Zhang W, Xiong YQ, Wu WZ, Wang L, Tang ZY, Sun HC (2008) High expression of macrophage colonystimulating factor in peritumoral liver tissue is associated with poor survival after curative resection of hepatocellular carcinoma. J Clin Oncol 26: $2707-2716$.

This work is published under the standard license to publish agreement. After 12 months the work will become freely available and the license terms will switch to a Creative Commons AttributionNonCommercial-Share Alike 3.0 Unported License.

Supplementary Information accompanies this paper on British Journal of Cancer website (http://www.nature.com/bjc) 